\title{
Factores contextuales del desarrollo infantil y su relación con los estados de ansiedad y depresión*
}

\section{Contextual factors of childhood development and its relation with anxiety and depression states}

\author{
Natalia Esparza \\ María Clara Rodríguez** \\ Universidad de la Sabana, \\ Bogotá, Colombia
}

Recibido: 25 de noviembre de 2008

Revisado: 20 de diciembre de 2008

Aceptado: 30 de enero de 2009

\section{Resumen}

Esta investigación tiene por objeto identificar los factores contextuales relacionados con los estados de ansiedad y depresión en niños escolarizados, valorados por sus docentes según la observación del comportamiento del niño en diversos contextos. Se trabajó con 254 niños entre 6 y 11 años. Se aplicaron la Escala Multidimensional de la Conducta de Reynolds \& Kamphaus (1992), que evalúa los problemas emocionales y de conducta. La Lista de Chequeo de Estresores (Jiménez \& Castro, 2006), que incluye Ambiente Físico, Relaciones Sociales, Exigencias Académicas y Entrevista de Evaluación y Caracterización. El análisis de regresión multivariada muestra que el factor exigencia académica se asocia con la presencia de estados de ansiedad, así como con relaciones sociales y exigencias académicas a los estados de depresión en la población infantil. El análisis correlacional indica una relación moderada entre la ansiedad y la depresión.

Palabras clave: ansiedad, depresión, niños en edad escolar, factores contextuales

* Esta investigación fue apoyada en su proceso de ejecución por la Universidad de la Sabana.

** Correspondencia: María Clara Rodríguez Díaz. Facultad de Psicología. Universidad de la Sabana. Correo electrónico: maria.rodriguez1@unisabana. edu.co. Dirección postal: Universidad de La Sabana, Puente del Común, Chía, Colombia. 


\section{Abstract}

This research aims to identify the contextual factors associated with states of anxiety and depression in children attending school, rated by their teachers according to the observation of the child's behavior in multiple contexts. The multidimensional behavioral scale of Reynolds \& Kamphaus (1992) that evaluate emotional and behavioral problems was applied along with stressors checking list (Jimenez y Castro, 2006) including physical environment, social relationships, academic requirements, evaluation interview and characterization to 254 children in school age between 6 an 11 years. Multivariate regression analysis shows that requirement academic factor is associated with anxiety states as well as social relationships and requirement academic related to depression states in children. Correlational analysis indicates a moderate relation between anxiety and depression.

Key words: anxiety, depression, elementary school aged children, contextual factors

\section{Introducción}

Durante gran parte del siglo XX, la investigación en el campo de la salud mental infantil se centró en comprender los estados afectivos y emocionales de los niños en las diferentes etapas del desarrollo. Lo anterior respondía a la prevalencia de los estados de ansiedad y depresión observados en diferentes ámbitos de interacción de los niños y jóvenes, tal como lo exponen González, Herrero, Viña, Ibáñez y Peñate (2004) y Rodríguez y Martínez (2001). Este interés responde a la alta prevalencia entre la población general y su impacto sobre el sufrimiento personal que producen. Según Bragado, Bersabé y Carrasco (1999), la ansiedad aparece como uno de los motivos de demanda asistencial más evidentes en los servicios de salud mental.

En este mismo sentido, en un estudio llevado a cabo con población infantil escolarizada del oriente antioqueño, se encontró que la prevalencia de sintomatología depresiva fue de 25,2\% (Vinaccia, 2006). En estudios realizados por Rivas, Vásquez y Pérez (1995), igualmente, la prevalencia de la ansiedad reportada por los maestros fue del $21,2 \%$ y del $26,5 \%$ para el caso de la depresión en la población infantil. Lo más importante de rescatar en esta situación es que el psicólogo clínico debe afrontar desde las estrategias de evaluación e intervención, la posibilidad de estar frente a la comorbilidad, aspecto que será discutido más adelante.
Si se tiene en cuenta que la dimensión emocional es fundamental dentro del desarrollo infantil, se pone en evidencia la necesidad de profundizar acerca de los diversos problemas emocionales que se pueden presentar a lo largo de la infancia, ya que éstos tienen un efecto importante sobre el desarrollo cognitivo, social y afectivo de los niños. Asimismo, son relevantes las consecuencias que se reflejan en etapas posteriores como la adolescencia e, incluso, en la adultez temprana, cuando estos problemas no son atendidos oportunamente tal como lo señalan Marsh y Graham (2001), quienes afirman que "existen continuidades a lo largo de la vida en muchas formas de psicopatología infantil. Aunque la naturaleza y gravedad de sus problemas puedan cambiar, muchos niños no superan las dificultades de la infancia” (p. 29).

Acero y Vásquez (2007) encontraron que aproximadamente el $20 \%$ de los niños y de los adolescentes en el mundo presentan un problema psicológico que afecta el bienestar y el desarrollo. Los autores especifican que las problemáticas más comunes son los trastornos de aprendizaje, por déficit de atención con hiperactividad, la depresión.

Esta tendencia ha sido confirmada por el estudio de salud mental, llevado a cabo por el Ministerio de Protección Social (2003), en el cual los trastornos de aparición más temprana son los trastornos de ansiedad y los problemas de conducta. Asimismo, el estudio reveló que los trastornos más comunes fueron los trastornos de ansiedad con una prevalencia del $19,3 \%$, los trastornos del estado 
de ánimo 15\% y los trastornos de uso de sustancias psicoactivas $(10,6 \%)$. Lo anterior da cuenta de la relevancia que adquiere el diagnóstico temprano en la vida de los niños, ya que es posible que éste se convierta en determinante de su estado psicológico futuro.

Las investigaciones relacionadas con la prevalencia en la población infantil de la ansiedad y la depresión no sólo ponen de manifiesto la importancia de reconocer que el diagnóstico forma parte de las estrategias de prevención, sino que también demuestran que la psicología deberá tener en cuenta que la variable edad de inicio, se constituye en un factor esencial a la hora de hacer pronósticos sobre la mejoría y sus efectos futuros. Específicamente, se reporta que para el caso de los trastornos de ansiedad, su aparición ocurre entre los seis y los veinticuatro años de edad, dependiendo del trastorno específico (Ministerio de Protección Social, 2003). Estos datos son congruentes con revisiones de la literatura en otras partes del mundo en las que se menciona que la mayor prevalencia de ansiedad en los niños se aprecia entre los seis y los dieciséis años de edad (Diez, 2000), lo que coincide con las etapas en las que más se reciben quejas por parte de padres y maestros. Esto pone en evidencia el impacto de la ansiedad sobre los entornos socio-afectivo y escolar.

La ansiedad se ha definido como una reacción adaptativa ante situaciones que representen o sean interpretadas como una amenaza o peligro para la integridad psicofísica del individuo, que actúa como un sistema de alerta ante situaciones potencialmente peligrosas. Ante la percepción de una situación considerada como amenazante, un "programa ansioso" es activado de forma refleja (Diez \& Sánchez, 2000; Bourne, 2000). Vale la pena aclarar que no necesariamente el estímulo generador de un estado de ansiedad debe ser externo, ya que en varios casos la ansiedad aparece sin fuente externa de amenaza reconocible (Belloch, Sandín \& Ramos, 1995). La conceptualización anterior pone de antemano la necesidad de realizar una distinción entre la ansiedad, como respuesta frente a un peligro real o potencial, la cual a un determinado nivel es benéfica, en tanto que cumple una función adaptativa como recurso fundamental de protección contra peligros físicos y sociales (Becerra et al., 2007).

Por otra parte, la que se conoce como ansiedad clínicamente significativa ha sido definida por Lazarus \& Averill (1972), como una emoción que surge a partir de la valoración del temor, incluyendo elementos simbólicos, anticipatorios e inciertos. Son precisamente los mecanismos cognitivos que median entre el estímulo estresante y la respuesta emocional los que permiten distinguir la ansiedad de otras emociones. Los planteamientos anteriores son congruentes con la propuesta de Barlow (1988), quien afirma que en la ansiedad existe la mediación de la estructura cognitivo-afectiva.

Si se parte de la importancia de analizar diversas dimensiones en la presentación de ansiedad, es posible ahora diferenciarla en función de la característica de la respuesta, es decir, si ésta es exagerada frente a estímulos amenazadores leves, reacciones que sólo se podrían dar frente a amenazas o situaciones graves. Así, de acuerdo con Díez (2000) existe un dominio emocional que incluye miedo, temores difusos, inseguridad, preocupaciones excesivas, anticipación del peligro de que algo va a pasar, interpretaciones amenazantes de las situaciones del entorno y una hipervigilancia ante estímulos que puedan representar amenazas potenciales; un dominio conductual con presencia de inquietud psicomotora, tendencia al llanto y conductas de evitación de objetos o situaciones que provocan miedo o ansiedad, así como una exageración de la respuesta de alarma.

Lo anterior es válido para comprender que en los niños, los dominios mencionados son congruentes con los planteamientos de Rodríguez y Martínez (2001), ya que en las cogniciones de los niños ansiosos hay una frecuencia excesiva de pensamientos negativos y catastrofización que afectan el desempeño cotidiano. En el orden social, los autores mencionan que los niños pueden presentar dificultades en la relación con pares y que sus compañeros pueden percibirlos como tímidos y aislados. Además, mencionan la posibilidad de presentar problemas escolares entre los cuales se destacan el rechazo escolar, el temor ante los 
exámenes y bajo rendimiento académico. Según el estudio, llevado a cabo por Jacques y Mash (2004), los niños con ansiedad tienden a preocuparse más por el futuro, por su bienestar y por las reacciones de otras personas.

En tal sentido, Roth y Heimberg (2006) encontraron que las personas que presentan ansiedad tienden a interpretar la información de forma negativa contribuyendo al mantenimiento de los estados ansiosos. Otros estudios han encontrado que en la población infantil son comunes las manifestaciones de angustia de forma preverbal, es decir, la evidencia de gestos de preocupación, gritos e hipertonía general (Pérez, León, Romo, Rodríguez \& Blanco, 1991).

Con referencia a la depresión, es bien sabido que el debate en torno a su presencia durante la infancia se ha extendido a lo largo del tiempo. En la actualidad aunque la depresión infantil es reconocida como un problema real y de alta incidencia en los niños, se podría decir que no existe un consenso real en cuanto a su conceptualización. Sin embargo, se mencionarán las perspectivas que por definición dominan el abordaje actual orientando el marco de referencia general que engloba esta problemática tan común entre la población infantil en la actualidad.

Según Méndez, Olivares y Ros (2001), y Weinber, Rutman, Sullivan, Pencik y Dietz (1973) la depresión se define como un problema psicológico complejo caracterizado por un estado de ánimo irritable, falta de motivación y disminución de la conducta adaptativa. Incluye, también, alteraciones del apetito, del sueño, de la actividad motora, cansancio, pobre autoconcepto, baja autoestima, sentimientos de culpa, dificultades para pensar o concentrarse, indecisión, ideas de muerte o suicidio e intentos de suicidio en la que los sistemas psicofisiológicos y motores son los más afectados. Los autores enfatizan en la repercusión negativa en los niveles personal (malestar físico, sufrimiento psicológico), familiar (deterioro de las relaciones padre-hijo), escolar, (descenso del rendimiento académico) y social (aislamiento).
En congruencia con estos estudios, Wicks e Israel (1997) establecen que la característica principal de la depresión es un estado de ánimo generalizado de infelicidad claramente subjetivo, lo que le da, en parte, el carácter clínico que posee. Las descripciones de niños y adolescentes que presentan depresión sugieren que suelen manifestar una pérdida de la experiencia de placer, retraimiento social, baja autoestima, incapacidad para concentrarse, trabajos escolares insuficientes, alteraciones en las funciones biológicas (dormir, comer, eliminación) y síntomas somáticos.

Estos resultados se ven apoyados por el estudio con población colombiana realizado por Vinaccia et al. (2006), en el que se encontró que los niños con depresión experimentaban un alto grado de sufrimiento subjetivo manifestado en la presencia del $25,2 \%$ de los menores con síntomas tales como el ánimo negativo, la anhedonia, la percepción de ineficiencia, los problemas interpersonales y la autoestima negativa. Asimismo, el estudio revela que existe una gran desmotivación por los contactos sociales, lo que genera disminución del refuerzo positivo en los niños.

Con base en las conceptualizaciones anteriores y apoyándonos en lo planteado por Belloch (1995) queda claro que en la depresión se observan diversos grupos de síntomas, desde anímicos, motivacionales y conductuales hasta la presentación de sintomatología física y manifestaciones en las relaciones interpersonales, que llevan a resaltar el grado de compromiso general que implica en la persona que la experimenta a considerar las consecuencias que un cuadro depresivo puede tener en la infancia.

Lo anterior ha sido corroborado en otras investigaciones en las cuales se plantea que la depresión infantil lleva a manifestaciones de irritabilidad, dificultades de aprendizaje, inseguridad, resistencia al juego, inasistencia a clases $y$, en general, problemas de comportamiento que se hacen evidentes en los diversos contextos del niño como el hogar y la escuela (Buela, Carretero \& De los Santos, 2001; Navarro, 2004). 
Las consideraciones anteriores son importantes, ya que éstas permiten al clínico comprender y explicar por qué en algunos casos los niños presentan problemas en sus diferentes entornos de interacción. Sin embargo, los avances en el conocimiento soportan la necesidad de situarnos de una manera menos desprevenida frente a estos niños y valorar la posibilidad de que podamos estar frente a un caso de comorbilidad.

Esto supone, como lo mencionan Vallejo, Gastó, Cardoner \& Catalán (2002), enfrentarnos a la posibilidad de que más de un diagnóstico se establezca en la misma persona bien sea de forma superpuesta o que se desarrollen durante el curso de alguno de ellos. Es así como a pesar de haber considerado durante largo tiempo la depresión y la ansiedad como entidades clínicas distintas, actualmente es fundamental reflexionar acerca de su alto índice de coexistencia, la cual es ahora entendida más como la norma que como la excepción.

De acuerdo con algunas valoraciones, entre un tercio y la mitad de los pacientes con uno de estos trastornos presentan síntomas del otro (Vallejo et al., 2002). De la misma forma, el estudio, realizado por González, et al., (2004), reveló que tres de cada cuatro casos diagnosticados con ansiedad presentan síntomas depresivos, lo cual puede también ocurrir al contrario. A su vez, se ha mostrado que los autorreportes sobre los niveles de ansiedad y depresión están altamente correlacionados con rangos entre 0,40 y 0,70 (Ollendick, Seligman, Goza, Byrd \& Singh, 2003).

Autores como Aláez, Martínez y Rodríguez (2000), así como Jacques y Mash (2004) encontraron que, de acuerdo con los autorreportes, los síntomas depresivos y los de ansiedad están altamente correlacionados, lo que sugiere un solapamiento entre los constructos depresión y ansiedad, fenómeno que puede se explicar debido a factores comunes como su etiología, factores de riesgo que comparten, así como limitaciones diagnósticas para diferenciarlas. Estos planteamientos son congruentes con la propuesta de González et al. (2004), quienes establecen que las razones que explican esta alta comorbilidad parecen ubicarse en la propia naturaleza de la ansiedad y la depresión, la cual hace que tiendan a cursar de forma conjunta.

Sin embargo, vale la pena mencionar que a pesar de la aparente claridad existente en torno a la presentación de ansiedad y depresión simultáneamente en gran cantidad de casos, persiste el debate en torno a si alguna de éstas dos se desarrolla primero que la otra. Otero (2000), uno de los autores que ha estudiado esta relación, comenta la alta frecuencia de niños con depresión que, a la vez, presentan síntomas de ansiedad, pero observa que hay pocos reportes acerca de la situación contraria, es decir, niños con ansiedad que presentan sintomatología depresiva. Esto implica enfrentarse a la posibilidad de que la presencia de un trastorno o problema pudiera anteceder a la aparición del otro, tal como el caso de la ansiedad que precede a la depresión (Marsh \& Graham, 2001).

Ante la ausencia de un consenso seguro acerca de la relación entre depresión y ansiedad y la situación de comorbilidad entre éstas, han surgido diversas posturas teóricas que han intentado ofrecer explicaciones claras acerca de la alta comorbilidad entre las dos. Actualmente, uno de los modelos imperantes en el abordaje de la comorbilidad existente entre ansiedad y depresión se conoce como el Modelo Tripartito postulado por Clark y Watson (1991) y aplicado por González et al. (2004), el cual es considerado como una ampliación del modelo de dos factores de Tellegan (Watson \& Tellegan, 1985), que planteaba la existencia de dos dimensiones del afecto independientes entre sí (afecto negativo y afecto positivo), con un patrón diferencial en sus relaciones con la depresión y la ansiedad.

El afecto negativo sería el factor común para ambas problemáticas, mientras que el afecto positivo se encontraría únicamente en la depresión. La ampliación propuesta por Clark y Watson (1991) incluye una tercera dimensión particular para la ansiedad, denominada activación fisiológica. El modelo posee una organización jerárquica en la que el afecto negativo se convierte en el factor de orden superior. 
En su estudio acerca de la aplicación del Modelo Tripartito de la ansiedad y la depresión en niños y adolescentes, Ollendick et al. (2003) mencionan la relevancia que ha ido adquiriendo dicho modelo en infantes de diversas edades. Luego de realizar un análisis factorial al modelo aplicado en niñas y niños, los autores sugieren la existencia de un factor individual tanto para la ansiedad como para la depresión, de forma que mientras la ansiedad se caracteriza por la tensión e hiperactivación fisiológica, en la depresión la característica principal es una ausencia de afecto positivo (Watson \& Tellegan, 1985).

Vale la pena aclarar que a pesar de una aparente concordancia entre los resultados acerca del Modelo Tripartito de la ansiedad y la depresión en la infancia, diversos autores (González et al., 2004; Jacques \& Mash, 2004) ponen de manifiesto la necesidad de tomar en consideración las variables género y edad (Chorpita, 2002). Asimismo, es importante recalcar que a pesar de los progresos en el entendimiento de la relación entre ansiedad y depresión a partir del Modelo Tripartito, la falta de investigaciones que soporten los hallazgos actuales hace que aún éste no sea un modelo explicativo claro y único para comprender cuestiones como la comorbilidad.

En este punto adquiere fundamental relevancia tomar en consideración el tema de la evaluación tanto de la ansiedad como de la depresión. Rodríguez y Martínez (2001) establecen que para el caso de la ansiedad, la evaluación debe ser muy cuidadosa e involucrar diversas estrategias, teniendo en cuenta los diferentes ambientes, las posibles personas que puedan brindar información sobre el niño y la comorbilidad con otros trastornos que se pueden presentar, priorizando el que puede ser la base y el agente de mantenimiento de la problemática. Esto contribuye a identificar el tipo de ayuda que los niños y adolescentes necesitan, y programar adecuadamente el plan de tratamiento.

Finalmente, considerar los aspectos que contribuyen al desarrollo y mantenimiento de la ansiedad y la depresión permitirá tener un marco amplio de comprensión y, a la vez, establecer los derroteros para intervenir de forma adecuada y oportuna. La influencia del temperamento, específicamente la inhibición conductual, ha sido considerada como un factor de riesgo para el caso de la ansiedad (Diez \& Sánchez, 2000). Otro aspecto como la presencia de ansiedad en los padres ha sido otra evidencia que aporta a la comprensión de la predisposición.

Lo anterior se relaciona con el concepto de ansiedad-rasgo que se refiere precisamente a esa inhibición comportamental y que se asocia con una mayor condicionabilidad a los estímulos aversivos (Rodríguez \& Martínez, 2001). Asimismo, se ha encontrado que existe la posibilidad de una vulnerabilidad genética, en cuanto diversos hallazgos, demuestran que niños con padres que presentan algún cuadro o trastorno de ansiedad tienden a presentar con mayor probabilidad ansiedad en algún momento de sus vidas.

Adicionalmente, el factor atencional podría influir debido a que ciertas personas con ansiedad parecen presentar dificultades en cuanto a focalización de estímulos congruentes con la interpretación del peligro, de forma que estas personas tienden a focalizarse en los estímulos amenazantes permanentemente (Mc Leod, Mathews \& Tata, 1986). Estos hallazgos son congruentes con los planteamientos de Rodríguez y Martínez (2001), quienes mencionan que una tendencia generalizada a centrarse en estímulos aversivos puede generar la presencia persistente de ansiedad. Por otro lado, el estilo cognitivo del niño también ha sido considerado por diversos autores (Rodríguez \& Martínez, 2001; Wicks \& Israel, 1997) como un factor de predisposición, por cuanto aquellos infantes que poseen un estilo centrado en la preocupación parecen estar en mayor riesgo de presentar ansiedad.

En cuanto a factores de adquisición de la ansiedad, Rodríguez y Martínez (2001) establecen que la teoría del condicionamiento clásico se ha constituido como una de las propuestas más importantes aplicadas a la población infantil, de la misma forma que lo ha sido la teoría del condicionamiento operante como factor de mantenimiento en dicha población. En general, estos modelos 
están basados en el supuesto de que los niños con temor o ansiedad excesiva tienen dificultad para controlar la presencia de cogniciones negativas, las cuales son irracionales y catastróficas (Rodríguez \& Martínez, 2001).

De la misma manera, se ha establecido que los acontecimientos vitales juegan un papel relevante en el mantenimiento de la ansiedad (Moreno, Del Barrio \& Mestre, 1995) debido a que tal como Becerra, et al. (2007) lo afirman, la presencia de un conflicto se relaciona con la aparición de estados ansiosos.

Con respecto a la depresión infantil, Méndez et al. (2001) mencionan que ésta es un problema complejo en el cual intervienen de forma conjunta factores de tipo ambiental, biológico, personal y psicológico. En el nivel biológico se ha relacionado un déficit funcional de serotonina con la aparición de la depresión, lo que aumenta la vulnerabilidad de la persona. Asimismo, Wicks e Israel (1997) señalan la influencia genética de forma que la probabilidad en la presentación de depresión en la infancia es más alta en niños con padres que presenten problemas o trastornos emocionales.

Sin embargo, los factores contextuales y situacionales para el caso de la ansiedad juegan un papel relevante en la manifestación o no de esta vulnerabilidad genética (Méndez et al., 2001; Bragado et al., 1999). Se han identificado diferentes factores predisponentes entre los que se destacan la falta de soporte social, condiciones sociodemográficas como el bajo nivel socioeconómico, la presencia de amenazas, los estilos de afrontamiento, la ausencia de una afectividad positiva y consistente por parte de los padres, los conflictos entre pares y figuras de autoridad, al igual que una autoridad parental inconsistente (Catalán, 2000). En esta misma dirección, Navarro (2004) señala la influencia de la calidad de las relaciones familiares tanto de los padres con los hijos como las relaciones maritales.

Estudios realizados con respecto a las pautas de crianza por Arvelo (2003) y Builes, Múnera, Salazar y Schnitter (2006) revelan el papel de éstas en el estado emocional, así como relaciones en- tre bajos niveles de reforzamiento a los hijos y la presentación de estados depresivos en ellos. El bajo nivel de refuerzo positivo explica la poca actividad del niño con depresión, cuya conducta adaptativa está sometida a un programa de extinción, dado que las personas evitan el contacto y lo someten a un aislamiento social involuntario. Esta situación es análoga a la pérdida de la fuente de refuerzo positivo como es la muerte de uno de los padres (Méndez et al. 2001).

Las demandas permanentes del entorno y sus implicaciones en el desarrollo del niño han suscitado un sinnúmero de preguntas sobre la forma en que los eventos estresores derivados de los contextos más inmediatos, como son la escuela y la familia, tienen implicaciones sobre el desarrollo emocional. En este sentido, Seiffge-Krenke (2001) resaltó la necesidad de definir los estresores relacionados con el colegio (evaluaciones, relaciones con pares, profesores, afectivas y cambios de grado) y con la familia (conflictos con padres), al tiempo que discutió el valor del afrontamiento como estrategia para lograr resolverlo desde el cambio de las condiciones que lo generan. Si bien existen numerosos estudios que muestran la influencia del estrés enfocado en los problemas derivados de la familia (Lemos, 2002), el colegio como contexto de desarrollo y en el cual el niño permanece gran parte del día, se considera un motivo más para llevar a cabo este trabajo.

El medio escolar supone condiciones positivas que aportan bienestar a los niños, sin embargo, tal y como se mencionó anteriormente, existen otras fuentes de problemas que plantean dificultades al niño entre las cuales el ambiente físico juega un papel importante. En este caso, por ejemplo, las aulas sobrepobladas, con excesos de ruido, con temperaturas no convenientes o con defectos de iluminación pueden afectar considerablemente el desempeño de los jóvenes (Orlandini, 1996).

En síntesis, se observa que varios factores están relacionados con la presencia de ansiedad y depresión en los niños en edad escolar. En la psicología, a pesar de reportar avances en varias direcciones, quedan abiertos algunos interrogantes en poblaciones particulares, lo cual da mayor im- 
portancia a este estudio que bien puede aportar elementos de interés para todos aquéllos que trabajan en la prevención de problemas emocionales en niños.

En consecuencia, los objetivos que guiaron este trabajo estuvieron relacionados con la identificación de los factores contextuales a la ansiedad y depresión en niños en edad escolar desde el modelo de riesgo y protección, su frecuencia de aparición y la relación existente entre estas dos problemáticas.

\section{Método}

\section{Diseño}

El presente estudio utilizó una metodología descriptiva correlacional, por cuanto ésta permite dar cuenta y analizar los diferentes factores asociados con un fenómeno particular (Hernández, Fernández \& Baptista, 2006). En este caso se tomaron en consideración los factores relacionados con el ámbito familiar y escolar, y su asociación con los estados de ansiedad y depresión.

\section{Participantes}

Se trabajó con un grupo de 254 niños escolarizados de estrato medio-bajo con edades comprendidas entre seis y once años, sus respectivos padres y catorce docentes. Los participantes fueron seleccionados de manera intencional y una vez conocieron el objetivo de la investigación dieron su consentimiento informado para participar.

\section{Instrumentos}

La Escala Multidimensional de la Conducta, desarrollada por Reynolds y Kamphaus (1992), evalúa problemas de conducta y trastornos emocionales, a la vez que permite identificar atributos positivos que pueden ser capitalizados en el proceso de tratamiento. Consta de tres cuestionarios, uno para padres, otro para docentes que recogen descripciones del comportamiento del niño en di- ferentes contextos y uno de autoevaluación para niños mayores de ocho años. Las escalas clínicas se puntúan de la siguiente manera: mayor a 90 "clínicamente significativo", entre 80 y 89 "alta" y entre 75 y 79 "riesgo". Esta prueba fue estandarizada en Medellín (Colombia), por el grupo de Neuropsicología y Conducta de la Universidad San Buenaventura; el grupo de Neurociencias de la Universidad de Antioquia, El Instituto Neurológico de Antioquia y la Universidad de Georgia. (Kamphaus et al., 2000).

Lista de Chequeo Percepción de Estresores consta de 41 situaciones que deben ser valoradas en una escala de 1. Nada; 2. Poco; 3. Bastante; 4. Mucho. Esta lista está conformada por seis escalas: rendimiento académico, en la que se incluyen las situaciones que hacen referencia a las consecuencias del bajo desempeño académico. Control aversivo, relacionada con sanciones o situaciones negativas que puedan afectar la conducta en el colegio. Relaciones con pares: situaciones que implican conflicto con los pares. Exigencia académica: situaciones que tienen relación con el tiempo y el nivel demandado por los deberes académicos. Conflictos con otros (dificultades con el profesor): dificultades en las interacciones con otros, diferentes a los pares dentro del colegio. Ambiente físico: está conformado por la percepción del espacio que hace parte del salón de clase donde permanece el estudiante (Jiménez \& Castro, 2006).

Entrevista de Evaluación y Caracterización: ésta se desarrolla tomando como base los datos que permiten la identificación de los niños y los padres, así como el registro de las condiciones relacionadas con la crianza, en las cuales es importante determinar la participación de los padres en ésta, así como en la consistencia en las normas. Por su parte, la organización familiar actual es importante también en la medida en que evalúa si el niño vive con su familia nuclear y extensa, así como las experiencias difíciles vividas por el niño en la infancia, tales como la separación, conflictos entre sus padres y la muerte de uno de ellos. Esta es diligenciada conjuntamente con los padres o el cuidador del niño. 


\section{Procedimiento}

Una vez establecido el contacto con las instituciones, las cuales se seleccionaron por conveniencia, se procedió a presentar el proyecto a directivos, docentes y padres quienes firmaron el consentimiento informado. Posteriormente, los padres evaluaron los factores relativos al entorno familiar y las características demográficas fueron otro insumo que nutrió este trabajo. Durante 1 año los docentes trabajaron en un proyecto de prevención que buscó optimizar su rol en el ámbito escolar como agente promotor en salud mental. Desde esta perspectiva, los docentes respondieron a la Escala Multidimensional de la Conducta para cada niño de la clase, ya que ellos habían sido preparados para identificar problemas de conducta que pueden aparecer súbitamente o, por el contrario, ser consistentes en un periodo de tiempo particular. Paralelamente, los niños identificaron los factores relativos al entorno escolar que pueden generar estrés.

\section{Resultados}

Los datos obtenidos a partir de la evaluación diagnóstica realizada por los docentes, fueron analizados a través del modelo multivariado de regresión logística que permite establecer el modelo que mejor predice la presencia de estados de depresión y ansiedad en los niños en edades comprendidas entre seis y once años.

En la tabla 1 se presentan los datos sociodemográficos de la población infantil que participó en el estudio. El $46 \%$ de la muestra fue constituida por niños mientras que el $53 \%$ restante lo constituyeron participantes del género femenino. La media de edad fue de 8,4 años para una mayoría de niños de diez años seguida de nueve, siete, ocho, seis y once años. El mayor porcentaje de participación se agrupa en el nivel de quinto grado de primaria, mientras que los grupos restantes oscilan entre $16,5 \%$ y $18,1 \%$.

Entre las características familiares se observa que el $70 \%$ de los niños conviven con su familia nuclear, el $30 \%$ con uno de sus padres, el $19 \%$ son hijos únicos y el 33\% han experimentado situaciones críticas, como por ejemplo la separación o muerte de uno de los padres, entre otras.

Tabla 1. Datos sociodemográficos de la población infantil del estudio

\begin{tabular}{lcc}
\hline & Frecuencia & $\%$ \\
\hline Género & & \\
Masculino & 118 & $46,5 \%$ \\
Femenino & 136 & $53,5 \%$ \\
Edad (años) & 8,45 & \\
media & & \\
6 & 40 & $15,7 \%$ \\
7 & 41 & $16,1 \%$ \\
8 & 40 & $15,7 \%$ \\
9 & 53 & $20,9 \%$ \\
10 & 56 & $22.0 \%$ \\
11 & 24 & $9,4 \%$
\end{tabular}

\section{Grado escolar} (Primaria)

$\begin{array}{lll}\text { Primero } & 43 & 16,9 \% \\ \text { Segundo } & 42 & 16,5 \% \\ \text { Tercero } & 46 & 18,1 \% \\ \text { Cuarto } & 45 & 17,7 \% \\ \text { Quinto } & 78 & 30,7 \%\end{array}$

\section{Características} familiares

$\begin{array}{lcc}\text { Familia nuclear } & 178 & 70,0 \% \\ \text { Padres separados } & 76 & 30,0 \% \\ \text { Hijos únicos } & 50 & 19,7 \% \\ \begin{array}{l}\text { Experiencia } \\ \text { traumática }\end{array} & 84 & 33,0 \%\end{array}$

Con base en los datos de evaluación obtenidos por parte de los docentes que participaron en el estudio, y tomando las puntuaciones que oscilan entre 75 y 90 , así, de 75 a 79 de riesgo, de 80 a 89 de alta y mayor de 90 , clínicamente significativo, 
142 niños presentan indicadores de ansiedad, lo cual señala una prevalencia del 55,9\% dentro de la población infantil del presente estudio. La conceptualización de la ansiedad de la cual partieron los docentes incluyó: estar nervioso, con miedo y preocupado por problemas reales o imaginarios.

De igual manera, se reportaron 132 casos con indicadores de depresión, lo cual señala una prevalencia del $52 \%$. Para los docentes, los sentimientos de tristeza, infelicidad y estrés que pueden resultar en la incapacidad de llevar a cabo actividades cotidianas sirvieron de marco para esta evaluación. En relación con el porcentaje de co-ocurrencia entre ansiedad y depresión, en el $39,8 \%$ de los casos se presenta la comorbilidad.

En la tabla 2 se pueden observar los factores asociados a la ansiedad, tanto en niños como en niñas. Se encuentra que los estresores por exigencia académica constituyen la variable que mejor explica la presencia de ansiedad en la población infantil. Dado que el riesgo relativo está cercano a $1(1,017)$ no es una variable que explique de manera robusta la presencia de ansiedad. Es decir, a pesar de existir una relación, esta ausencia de fortaleza está dada por las características del modelo, que podría irse afinando en el futuro.

Tabla 2. Factores asociados a la presentación de ansiedad en la población infantil total

\section{Variables en la ecuación}

\begin{tabular}{|c|c|c|c|c|c|c|c|c|c|}
\hline & & \multirow[b]{2}{*}{ B } & \multirow[b]{2}{*}{ E.T. } & \multirow[b]{2}{*}{ Wald } & \multirow[b]{2}{*}{ Gl } & \multirow[b]{2}{*}{ Sig. } & \multirow[b]{2}{*}{$\operatorname{Exp}(B)$} & \multicolumn{2}{|c|}{$\begin{array}{l}\text { I.C. } 95.0 \% \text { para } \\
\operatorname{EXP(B)}\end{array}$} \\
\hline & & & & & & & & Inferior & Superior \\
\hline $\begin{array}{c}\text { Paso } \\
1\end{array}$ & $\begin{array}{l}\text { Estresores } \\
\text { por exigencia } \\
\text { académica }\end{array}$ & 0,017 & 0,005 & 13,671 & 1 & 0,000 & 1,017 & 1,008 & 1,026 \\
\hline & Constante & $-1,263$ & 0,246 & 26,344 & 1 & 0,000 & 0,283 & & \\
\hline
\end{tabular}

En la tabla 3 se muestran los factores asociados a la ansiedad en las niñas. Se observa que las variables que explican la presencia de ansiedad son los estresores de exigencia académica, lo que aumenta en un 1,03 la probabilidad de exhibir in- dicadores de ansiedad. Por el contrario, al aplicar el modelo en niños no se encuentran factores particulares para el género masculino asociados a la ansiedad.

Tabla 3. Factores que predicen la presencia de ansiedad en niñas

\section{Variables en la ecuación}

I.C. $95.0 \%$ para

$\operatorname{EXP}(B)$

B E.T. Wald Gl Sig. $\operatorname{Exp(B)}$ Inferior Superior

Paso Estresores por

1 exigencia académica

$0,025 \quad 0,006$

17,165

$1 \quad 0,000$

1,026

1,013

1,038

$-0,998$

0,302


En la tabla 4 se observan los factores asociados a la presentación de indicadores de depresión en la población infantil del estudio. Se observa que los estresores en las relaciones con pares y conflictos con otros son las variables que mejor predicen la presentación de depresión en los niños, lo que indica que la presencia de estos dos factores aumenta en 2,5 veces la probabilidad de aparición de depresión frente a los niños que no están expuestos a este tipo de dificultades. Lo anterior pone de manifiesto que para el caso de la depresión, el modelo utilizado es mucho más robusto para explicar la presencia de estas problemáticas.

Tabla 4. Factores asociados con la depresión en la población infantil

\begin{tabular}{|c|c|c|c|c|c|c|c|c|c|}
\hline \multicolumn{10}{|c|}{ Variables en la ecuación } \\
\hline & & \multirow[b]{2}{*}{ B } & \multirow[b]{2}{*}{ E.T. } & \multirow[b]{2}{*}{ Wald } & \multirow[b]{2}{*}{ gl } & \multirow[b]{2}{*}{ Sig. } & \multirow[b]{2}{*}{$\operatorname{Exp}(B)$} & \multicolumn{2}{|c|}{$\begin{array}{c}\text { I.C. } 95.0 \% \text { para } \\
\operatorname{EXP(B)}\end{array}$} \\
\hline & & & & & & & & Inferior & Superior \\
\hline \multirow[t]{2}{*}{$\begin{array}{c}\text { Paso } \\
1^{\text {a }}\end{array}$} & $\begin{array}{l}\text { Estresores en rela- } \\
\text { ciones con pares }\end{array}$ & 0,033 & 0,006 & 27,725 & 1 & 0,000 & 1,034 & 1,021 & 1,047 \\
\hline & Constante & $-2,310$ & 0,323 & 51,070 & 1 & 0,000 & 0,099 & & \\
\hline \multirow{3}{*}{$\begin{array}{l}\text { Paso } \\
2^{\mathrm{b}}\end{array}$} & Conflictos con otros & 0,893 & 0,426 & 4,392 & 1 & 0,036 & 2,442 & 1,059 & 5,630 \\
\hline & $\begin{array}{l}\text { Estresores en } \\
\text { relaciones con } \\
\text { pares }\end{array}$ & 0,033 & 0,006 & 27,107 & 1 & 0,000 & 1,034 & 1,021 & 1,047 \\
\hline & Constante & $-2,463$ & 0,338 & 53,237 & 1 & 0,000 & 0,085 & & \\
\hline \multicolumn{10}{|c|}{$\begin{array}{l}\text { a. Variable(s) introducida(s) en el paso 1: Estresores en } \\
\text { relaciones sociales. }\end{array}$} \\
\hline \multicolumn{10}{|c|}{$\begin{array}{l}\text { b. Variable(s) introducida(s) en el paso 2: Dificultades } \\
\text { con el profesor. }\end{array}$} \\
\hline
\end{tabular}

En relación con los factores asociados con depresión infantil no se encuentran variables específicas para el género masculino. En la tabla 5 se muestran los factores que mejor predicen la presencia de depresión en las niñas. Se observa que al igual que en la población infantil general, los estresores en las relaciones con pares así como los conflictos con otros, aumentan 13,83 veces más la probabilidad en las niñas de presentar depresión.

La tabla 6 muestra los factores que podrían proteger a los niños frente a la posibilidad de desarrollar síntomas de ansiedad. Los resultados indican que el ser hijo único se constituye como un factor protector frente a la ansiedad infantil. 
Tabla 5. Factores asociados con la depresión en las niñas

\section{Variables en la ecuación}

\section{I.C. $95.0 \%$ para $\operatorname{EXP(B)}$}

\begin{tabular}{|c|c|c|c|c|c|c|c|c|c|}
\hline & & B & E.T. & Wald & $\mathrm{Gl}$ & Sig. & $\operatorname{Exp}(B)$ & Inferior & Superior \\
\hline \multirow[t]{2}{*}{$\begin{array}{c}\text { Paso } \\
1 a\end{array}$} & $\begin{array}{l}\text { Estresores en } \\
\text { relaciones } \\
\text { con pares }\end{array}$ & 0,049 & 0,009 & 28,216 & 1 & 0,000 & 1,050 & 1,031 & 1,069 \\
\hline & Constante & $-2,275$ & 0,417 & 29,781 & 1 & 0,000 & 0,103 & & \\
\hline \multirow[t]{3}{*}{$\begin{array}{l}\text { Paso } \\
2 b\end{array}$} & $\begin{array}{l}\text { Conflictos } \\
\text { con otros }\end{array}$ & 2,578 & 0,781 & 10,909 & 1 & 0,001 & 13,171 & 2,853 & 60,813 \\
\hline & $\begin{array}{l}\text { Estresores en } \\
\text { relaciones } \\
\text { con pares }\end{array}$ & 0,054 & 0,011 & 25,780 & 1 & 0,000 & 1,055 & 1,034 & 1,078 \\
\hline & Constante & $-2,798$ & 0,504 & 30,790 & 1 & 0,000 & 0,061 & & \\
\hline
\end{tabular}

a. Variable(s) introducida(s) en el paso 1: Estresores en relaciones sociales

b. Variable(s) introducida(s) en el paso 2: Dificultades con el profesor

Tabla 6. Factores protectores a la ansiedad en los niños

\section{Variables en la ecuación}

\begin{tabular}{|c|c|c|c|c|c|c|c|c|c|}
\hline \multirow[b]{3}{*}{$\begin{array}{c}\text { Paso } \\
1\end{array}$} & & \multirow[b]{2}{*}{ B } & \multirow[b]{2}{*}{ E.T. } & \multirow[b]{2}{*}{ Wald } & \multirow[b]{2}{*}{$\mathrm{gl}$} & \multirow[b]{2}{*}{ Sig. } & \multicolumn{3}{|c|}{$\begin{array}{l}\text { I.C. } 95.0 \% \text { para } \\
\text { EXP(B) }\end{array}$} \\
\hline & & & & & & & (B) & Inferior & Superior \\
\hline & Hijo único & $-19,668$ & 9220,900 & 0,000 & 1 & 0,998 & 0,000 & 0,000 & - \\
\hline & Constante & $-1,535$ & 0,295 & 27,153 & 1 & 0,000 & 0,215 & & \\
\hline
\end{tabular}

B. Se ha determinado un procedimiento por pasos, ya que al eliminar la variable menos significativa se obtuvo un modelo previamente ajustado

A fin de analizar la posible comorbilidad entre la ansiedad y la depresión infantil, se realizó un análisis de correlación de Spearman, el cual aparece en la tabla 7. Los resultados señalan que existe una correlación entre ansiedad y depresión identificada por los docentes de 0,59 con una $\mathrm{p} \leq \mathrm{a}$ 0,00 , lo que indica una correlación moderada. 
Tabla 7. Correlación entre la ansiedad y la depresión infantil

\begin{tabular}{|c|c|c|c|c|}
\hline & & & $\begin{array}{l}\text { Depresión } \\
\text { (Cl-D) }\end{array}$ & $\begin{array}{l}\text { Ansiedad } \\
\text { (Cl-D) }\end{array}$ \\
\hline \multirow[t]{6}{*}{$\begin{array}{l}\text { Rho de } \\
\text { Spearman }\end{array}$} & Depresión (Cl-D) & $\begin{array}{l}\text { Coeficiente de } \\
\text { correlación }\end{array}$ & 1,000 & $0,589^{* *}$ \\
\hline & & Sig. (bilateral) & & 0,000 \\
\hline & & $N$ & 254 & 254 \\
\hline & Ansiedad (Cl-D) & $\begin{array}{l}\text { Coeficiente de } \\
\text { correlación }\end{array}$ & $0,589^{* *}$ & 1,000 \\
\hline & & Sig. (bilateral) & 0,000 & . \\
\hline & & $N$ & 254 & 254 \\
\hline
\end{tabular}

** La correlación es significativa al nivel 0,01 (bilateral).

* La correlación es significativa al nivel 0,05 (bilateral).

\section{Discusión}

Los datos correspondientes a la prevalencia, tanto de la ansiedad como de la depresión, revelan una incidencia importante en la población infantil; esto es consistente con los hallazgos de Aláez (2000) y Bragado et al., (1999) se confirma la alta prevalencia de la ansiedad y la depresión en esta población.

Sin embargo, en este estudio se denota una mayor tasa de prevalencia para los indicadores de ansiedad y depresión, frente a otros problemas psicológicos más comunes en los niños. En nuestro contexto, estos resultados son consistentes al compararlos con los de Vinaccia et al. (2006), especialmente en el caso de la depresión. Se deberán tomar con cautela, en el sentido en que si bien la tasa es alta, ésta puede corresponder a la ampliación en el intervalo de valoración de los puntajes como significativos. El rango evaluado incluyó los puntajes de 75 a 79 de "riesgo", de 80 a 89 , alto, y mayor de 90 , como clínicamente significativo.

Esta ampliación de rango hace que la valoración incluya a todos los niños que muestren signos emocionales de miedo, preocupación o nervio- sismo, para el caso de ansiedad. Igualmente, se aplica para el caso de la tristeza, infelicidad o estrés. Lo anterior supone discutir y entender los resultados, desde una perspectiva de estado, que es transitorio y reactivo a una situación particular. De hecho, la asociación a unas condiciones contextuales particulares, como las relacionadas con las exigencias del entorno desde el punto de vista académico y social fueron relevantes. Desde el paradigma de riesgo y protección, éstas pueden ser modificadas, atendiendo a los lineamientos de prevención.

En este sentido, los docentes, quienes habían sido preparados para abordar desde su función el rol de agente promotor en salud mental, afinaron su pauta de observación, lo que no significa que se ha patologizado este grupo de niños observados, por el contrario, indica que este mecanismo puede permitir prever con anticipación una serie de dificultades que en el futuro se pudieran presentar.

Estos resultados muestran, tal como lo afirman Rodríguez y Martínez (2001), que la exposición a estímulos ambientales estresantes se puede convertir en factor predisponente para desarrollar un problema emocional, en especial cuando existe cierta vulnerabilidad (Diez \& Sánchez, 2000). Te- 
niendo en cuenta que en la presentación de ansiedad pueden entrar en juego una combinación de diversos factores de tipo biológico y socioambiental, se encuentra que, para el caso de la población infantil, factores como la exposición a estresores por exigencia académica se asocian con la presentación de estados de ansiedad.

Una posible explicación a la asociación entre estresores de exigencia académica y ansiedad podría radicar en los planteamientos de Rodríguez \& Martínez (2001) y Wicks \& Israel (1997), quienes mencionan que los niños que poseen un estilo cognitivo centrado en la preocupación, tienen mayor predisposición a desarrollar ansiedad de forma que una excesiva inquietud por la situación académica podría llevar a estados ansiosos en los niños escolarizados. Asimismo, es probable que, por ejemplo la inseguridad, como característica inherente a esta problemática (Diez, 2000), dificulte el afrontamiento de situaciones estresantes. Lo anterior pone en evidencia el hecho de que estas situaciones pueden llevar a afectar el nivel de desempeño académico.

En el estudio llevado a cabo por Rivas et al. (1995) se destaca la presencia de variables escolares, tales como el rendimiento académico, asociadas de manera significativa con problemas psicológicos infantiles. Estos planteamientos son consistentes con las afirmaciones de Barraza (2006), quien establece que "el inicio de la escolarización y su posterior desarrollo, sin olvidar el tránsito de un nivel educativo a otro, suelen ser eventos estresantes para el ser humano que en ese momento juega el rol de alumno" (p. 4).

De igual forma, situaciones como la evaluaciones a las que los niños se ven expuestos, se constituyen también en un estresor académico (Barraza, 2006) lo que recalca cómo la presencia de estresores de exigencia académica efectivamente podrían predecir la presentación de estados de ansiedad. Esta afirmación se ve apoyada en los planteamientos de Pellicer, Salvador y Benet (2002), quienes expresan que la exposición a los exámenes académicos es una de las situaciones potencialmente estresantes más importantes a las que se enfrentan los estudiantes.
En su revisión acerca del agotamiento emocional en niños y jóvenes escolarizados, Garcés (1995) manifiesta que al estar expuestos a altas exigencias escolares que se deben cumplir, los niños en etapa escolar pueden llegar a percibir falta de control sobre el ambiente escolar, lo que genera frustración, apatía y angustia. Igualmente, menciona que en el ámbito escolar se puede imponer sobrecarga en relación con las exigencias, generando lo que se ha conceptualizado como el agotamiento emocional escolar, el cual se ha relacionado con fenómenos como el fracaso escolar, fobia escolar y depresión.

Los hallazgos obtenidos en la presente investigación pueden ser apoyados también por los resultados obtenidos en el estudio de Oros y Vogel (2005), quienes comentan la asociación entre estresores académicos y la presentación de ansiedad. En dicho estudio, la preocupación por las relaciones con pares también aparece como uno de los estresores relacionados con la aparición de ansiedad.

Estos resultados confirman el hecho de que los estresores de tipo académico son los factores que mejor predicen la presentación de ansiedad en la población infantil. Sin embargo, los datos muestran que los estresores de tipo social guardan una relación más cercana con la depresión y la tristeza. Baker, Grant y Morlock (2008) confirman estos resultados al evaluar la influencia de las características de la relación profesor-alumno, como la cohesión, la calidez y sus implicaciones en el rendimiento escolar y a la vez sobre la adaptación del niño con problemas emocionales.

En este sentido, los sentimientos de infelicidad, tristeza y estrés relacionados con las dificultades con otros, entre ellos el profesor, ponen en evidencia el sufrimiento psicológico mencionado por Méndez et al. (2001), Vinaccia et al. (2006) y Wicks e Israel (1997), así como el malestar emocional relacionado con el miedo y la preocupación. El estrés, proveniente de la mala relación, con el profesor tiene implicaciones motivacionales importantes en la medida en que esto representa una pérdida de refuerzos positivos, restricción que incide sobre el desarrollo y mantenimiento de la depresión (Vinaccia et al., 2006). 
En otros estudios, como el de Méndez et al. (2001), se tiende a interpretar este conflicto como un evento que suscita falta de soporte social y disminución del refuerzo positivo. Esta carencia apoya los resultados obtenidos, puesto que tal carencia genera mayor estrés en los niños y, a la vez, afecta su nivel de respuesta frente a situaciones difíciles.

Por su parte, a diferencia de otras investigaciones (Navarro, 2004; Catalán, 2000), los datos de la presente investigación muestran que el tipo de organización familiar en este grupo de participantes, más que predecir problemas de tipo emocional, se podría considerar un aspecto que no hace vulnerable a los niños, tanto como los estresores provenientes del ámbito escolar. Al respecto, Oros y Vogel (2005) plantean:

Los estresores de un niño en edad escolar se situarán predominantemente en el contexto escolar y la interacción con los pares [...] en el área escolar sobresalen el cambio de escuela, el cambio de ciclo, la repetición de grado, el cambio de profesor, el aumento de trabajo y los plazos en tres o más asignaturas (pp. 88 89).

Otros estresores académicos importantes son las discusiones con profesores, sentirse solo, insatisfacción con su imagen entre otras variables. Estos aspectos resultan especialmente importantes si se tiene en cuenta que al analizar la presencia de factores asociados comunes a los estados de depresión y ansiedad, este estudio revela que factores como la exposición a estresores de exigencia académica (asociados con la ansiedad) y dificultades con otros (relacionados con la depresión) se podrían conceptualizar como variables similares, por cuanto es posible que niños expuestos a situaciones de exigencia académica presenten problemas con sus maestros. Esto llevaría a plantear factores comunes asociados a la presentación de ansiedad y depresión.

En su estudio, Oros y Vogel (2005) indican que los estresores sociales presentan una prevalencia en la población infantil del $25,7 \%$, mientras que existe una prevalencia del $12,9 \%$, en relación con estresores académicos. Estos resultados se relacionan con los obtenidos en el presente estudio, los cuales confirman el impacto que sobre la población infantil generan los estresores académicos y sociales, que se convierten en factores que podrían estar asociados con la presentación de problemas emocionales tales como la ansiedad y la depresión. Lo anterior, pone de manifiesto la necesidad de reflexionar acerca del valor que poseen los contextos escolar y social del niño sobre su desarrollo emocional.

En este sentido, la prevención se tendrá que articular en dos escenarios vitales que garanticen la optimización de los recursos de afrontamiento de los niños, frente a situaciones que son consideradas adversas. Se destaca la importancia de promover relaciones saludables entre pares y especialmente con sus profesores, en la medida en que su cercanía y cohesión permiten articular acciones a favor de rescatar las fortalezas de los niños en el aula. Por otro lado, las pautas de crianza y la organización familiar, que fueron factores estudiados en este trabajo, muestran que el modelo no permitió predecir la dirección de su relación. Así, en el futuro se continuará profundizando sobre este aspecto, de tal manera que se puedan articular estos resultados y ampliar la participación de los diferentes actores en el contexto de desarrollo de los niños.

La evaluación de los docentes, si bien no es el único camino para determinar la presencia de indicadores emocionales, sí se constituye en una fuente de información valiosa para validar, ampliar y hacer seguimiento al niño, incluso entrar en diálogo con otros contextos de interacción, como la familia. La evaluación de los padres será otro insumo que deberá complementar estos resultados de investigación, a fin de afinar el modelo que mejor predice la presencia de estados de ansiedad y depresión, cuando existen diferentes fuentes de información.

La co-ocurrencia entre ansiedad y depresión es otro aspecto que ayudará a los docentes y a los padres a actuar oportunamente frente a estas problemáticas, de tal manera que no afecten su desarrollo ni su nivel de adaptación frente a los cambios propios de las condiciones del contexto actual. Tal como Clark y Watson (1991) plantean, 
la explicación a la co-ocurrencia entre la ansiedad y la depresión podría radicar en la presencia del afecto negativo como factor común para estos dos cuadros. En concordancia con estudios como el de Aláez et al., (2000), González et al., (2004) y Ollendick et al., (2003), en este estudio se evidencia la co-ocurrencia entre ansiedad y depresión aunque en un grado moderado, si se compara con otras investigaciones.

Para finalizar, es importante rescatar la variable género por sus implicaciones en esta investigación, dado que los datos muestran cómo en el caso de las niñas la sensibilidad frente a los estresores y su relación con la ansiedad y la depresión son muy relevantes. Bragado et al. (1999) ya advertían tasas diferenciales entre géneros. Vinaccia et al. (2006) no encontraron diferencias antes de los doce años de edad en población colombiana; sin embargo, se sigue argumentando la relación entre acontecimientos vitales estresantes y la adquisición de la ansiedad y la depresión. En la presente investigación, la ampliación en el rango criterio para el análisis, esto es, la inclusión de todos los puntajes correspondientes a niveles clínicamente significativos, altos y en riesgo, pudo haber aumentado el número de casos y, a la vez, impactar los datos de prevalencia.

Otra importante reflexión sobre estos hallazgos es que nos permiten, dentro de la línea de la prevención, pensar en que si el punto de intersección entre estas problemáticas es el afecto negativo como se mencionó anteriormente, padres y docentes tendrán la responsabilidad de observar el afecto del niño en los diferentes contextos de desarrollo y a lo largo del tiempo. El psicólogo en esta medida tendrá mayores oportunidades de obtener información de diversas fuentes de valoración que aportarán a la intervención oportuna, adecuada y coherente con los protocolos desarrollados para la atención desde la psicología clínica infantil.

No menos importante será continuar la formación de los docentes en el campo de la promoción en salud mental de manera concurrente con los padres de familia. En este sentido, el camino que hemos recorrido nos permite garantizar que la observación del comportamiento del niño en el aula, por parte de los docentes, de manera juiciosa y sistemática, aportará de manera permanente en la intención de estar cada vez más cerca de la prevención.

\section{Referencias}

Acero, A.R. \& Vásquez, R. (2007). Psiquiatría infantil en el hospital pediátrico. Revista Colombiana de Psiquiatría, 36 (3), 460-470.

Aláez, M. Martínez, R. \& Rodríguez, C. (2000). Prevalencia de trastornos psicológicos en niños y adolescentes, su relación con la edad y el género. Psicothema, 12 (4), 525-532.

Arvelo, L. (2003). Función paterna, pautas de crianza y desarrollo psicológico en adolescentes: implicaciones psicoeducativas. Acción Pedagógica, 12 (1), 20-30.

Baker, J.A. Grant, S. \& Morlok, L. (2008). The Teacher-Student Relationship as a Developmental Context of Children with Internalizing or Externalizing Behavior Problems. School Psychology Quarterly, 23 (1), 3-15.

Barlow, D.H. (1988). Anxiety and its Disorders: The Nature and Treatment of Anxiety and Panic. New York: Guilford.

Barraza, A. (2006, 21 de noviembre). Un modelo conceptual para el estudio del estrés académico. Recuperado el 20 de noviembre de: http://www.psicologíacientífica.com/bv/ psicología-167-1-un-modelo-conceptual-parael-estudio-del-estrés-académico.html.Psicología Científica.com .

Becerra, A.M., Madalena, A.C., Estanislau, C., Rodríguez, J.L., Dias, H., Bassi, A., Chagas, D.A. \& Morato, S. (2007). Ansiedad y miedo: su valor adaptativo y maladaptaciones. Revista Latinoamericana de Psicología, 39 (1), 75-81.

Belloch, A., Sandín, B. \& Ramos, F. (1995). Manual de psicopatología (volumen I). Madrid: MC. Graw Hill. 
Bragado, C., Bersabé, R. \& Carrasco, I. (1999). Factores de riesgo para los trastornos conductuales, de ansiedad, depresivos y de eliminación en niños y adolescentes. Psicothema, 11 (4), 939-956.

Bourne, E.J. (2000). The Anxiety and Phobia Workbook ( $3^{\text {rd }}$ edition). New York: New Harbinger Publications.

Buela, G., Carretero, H. \& De los Santos, M. (2001). Relación entre la depresión infantil y el estilo de respuesta reflexivo-impulsivo. Salud Mental, 24 (3), 17-23.

Builes, L.D., Múnera, P.C., Salazar, C.J. \& Schnitter, M. (2006). El encuentro de los desencuentros: pautas de crianza presentes en las familias de niños menores de la calle institucionalizados. Informes Psicológico, 8, 57-667.

Catalán, R. (2000). Factores psicosociales. En Vallejo, J. \& Gastó, C. (Eds). Trastornos afectivos: ansiedad y depresión. Barcelona: Masson.

Chorpita, B.F. (2002). The Tripartite Model and Dimensions of Anxiety and Depression: An Examination of Structure in a Large School Sample. Journal of Abnormal Psychology, 30, 177-190.

Clark, L.A. \& Watson, D. (1991). Tripartite Model of Anxiety and Depression: Psychometric Evidence and Taxonomic Implications. Journal of Abnormal Psychology, 100, 316-336.

Diez, C. (2000). Clínica. En Vallejo, J. \& Gastó, C. (Eds). Trastornos afectivos: ansiedad y depresión. Barcelona: Masson.

Diez, C. \& Sánchez, L. (2000). Trastornos médicos En J. Vallejo, \& C. Gastó, (Eds). Trastornos afectivos: ansiedad y depresión. Barcelona: Masson.

Ministerio de Protección Social (2003). Estudio Nacional de Salud Mental. Recuperado el 1 de febrero de 2007 de: www.miniproteccionsocial.gov.co/vbccontent/newsdetcil. asp?|D=14822\& IDcompany=3
Garcés, E. (1995). Bornout en niños y adolescentes: un nuevo síndrome en psicopatología infantil. Psicothema, 7 (1), 33-40.

González, M., Herrero, M., Viña, C.M., Ibañez, I. \& Peñate, W. (2004). El modelo tripartito: relaciones conceptuales y empíricas entre ansiedad, depresión y afecto negativo. Revista Latinoamericana de Psicología, 36 (2), 289-304.

Hernández, R., Fernández, C. \& Baptista, P. (2006). Metodología de la investigación. México: Mc Graw Hill.

Jacques, H. \& Mash, E.J. (2004). A Test of the Tripartite Model of Anxiety and Depression in Elementary and High School Boys and Girls. Journal of Abnormal Psychology, 32 (1), 13-25.

Jiménez, I.A. \& Castro, C. (2006). Correlación entre percepción de estresores, estrategias de afrontamiento y estrés en jóvenes. Tesis de maestría en Psicología Clínica, Bogotá: Fundación Universitaria Konrad Lorenz.

Kamphaus, R.W., Jiménez, M.E., Pineda, D., Rowe, E.W., Fleckenstein, L., Restrepo, M.A., Puerta, I.C., Jiménez, I., Mora, O., Sánchez, J.L., García, A. \& Palacio, L.G. (2000). Análisis transcultural de un instrumento de dimensiones múltiples en el diagnostico del déficit de atención. Revista de Neuropsicología, Neuropsiquiatría y Neurociencias, 2 (2), 51-63.

Lazarus, R. \& Averill, J. (1972). Emotion and Cognition: With Special Reference to Anxiety En C. Splieberg (Ed.). Anxiety: Current Trends in Theory and Research (Volume II, 242-284). New York: Academic Press.

Lemos, M. (2002). Social an Emotional Processes in the Classroom Setting: a Goal Approach. Anxiety, Stress and Coping, 15 (4) 383-400.

Mc Leod, C., Mathews, A. \& Tata, P. (1986). Attentional Bias in Emotional Disorders. Journal of Abnormal Psychology, 95, 15-20. 
Marsh, E.J. \& Graham, S.A. (2001). Clasificación y tratamiento de la psicopatología infantil. En Caballo, V. \& Simon, M.A. Manual de Psicología Clínica Infantil y del Adolescente: trastornos generales. Madrid: Pirámide.

Méndez, F.X., Olivares, J. \& Ros, M.C. (2001). Estado de ánimo depresivo. Características clínicas y tratamiento de la depresión en la infancia y adolescencia. En Caballo, V. \& Simon, M.A. Manual de Psicología Clínica Infantil y del Adolescente: trastornos generales. Madrid: Pirámide.

Moreno, C., Del Barrio, V. \& Mestre, V. (1995). Ansiedad y acontecimientos en adolescentes. Revista Latinoamericana de Psicología, 27 (3), 471-496.

Navarro, M.E. (2004). Depresión en niños con trastorno por déficit de atención con hiperactividad. Reflexiones, 83 (1), 107-120.

Orlandini, A. (1996). El estrés: ¿qué es y cómo evitarlo? México: Fondo de Cultura Económica.

Ollendick, T.H., Seligman, L.D., Goza, A.B., Byrd, D.A. \& Singh, K. (2003). Anxiety and Depression in Children and Adolescents: A Factor Analytic Examination of the Tripartite Model. Journal of Child and Family Studies, 12 (2), 157-170.

Oros, V. \& Vogel, G. (2005). Eventos que generan estrés en la infancia: diferencias por sexo y edad. Enfoques, 17 (1), 85-101.

Otero, A. (2000). Historia familiar y genética de los trastornos afectivos. En Vallejo, J. \& Gastó, C. (Eds.) Trastornos afectivos: ansiedad y depresión. Barcelona: Masson

Pellicer, O.; Salvador, A. \& Benet, I. (2002). Efectos de un estresor académico sobre las respuestas psicológica e inmune en jóvenes. Psicothema, 14 (2), 317-322.

Pérez, M.A., Leon, M.R., Romo, M.L., Rodríguez, L. \& Blanco, A. (1991). Breve exposición sobre las diferentes manifestaciones ansiosas des- critas en la infancia. Anales de Psicología, 7 (1), 45-55.

Reynolds, C. \& Kamphaus, R.W. (1992). Behavior Assessment System for Children -BASC. Circle Pines: American Guidance Service.

Rivas, M., Vásquez, J.L. \& Pérez, M. (1995). Alteraciones psicopatológicas en la infancia. Psicothema, 7 (3), 513-526.

Rodríguez, M. \& Martínez, N.C. (2001). Trastorno de ansiedad generalizada y trastorno de pánico en niños y adolescentes. En Caballo, V. \& M.A. Simon, (Eds.) Manual de Psicología Clínica Infantil y del Adolescente: trastornos generales. Madrid: Pirámide.

Roth, D. \& Heimberg, R.G. (2006). Cognitive Vulnerability to Social Anxiety. Journal of Social and Clinical Psychology, 25 (7), 755-778.

Seiffge-Krenke, I. (2001). Predicting Adolescent Symptomatology through Stressful Events and Coping Style. Journal of Adolescence, 23, 675-692.

Vallejo, J., Gastó, C., Cardoner, N. \& Catalán, R. (2002). Comorbilidad de los trastornos afectivos. Barcelona: Ars Médica.

Vinaccia, S., Gaviria, A.M., Atehortúa, L.F., Martínez, P.H., Trujillo, C. \& Quiceno, J.M. (2006). Prevalencia de depresión en niños escolarizados entre 8 y 12 años del oriente antioqueño a partir del "Child Depression Inventory"-CDI. Diversitas Perspectivas en Psicología, 2 (2), 217-227.

Watson, D. \& Tellegan, A. (1985). Toward a Consensual Structure of Mood. Psychological Bulletin, 96, 219-235.

Weinber, W.A., Rutman, J., Sullivan, L., Pencik, E.C. \& Dietz, S.G. (1973). Depression in Children Referred to an Education Diagnostic Center. Journal of Pediatric, 83, 1065-1072.

Wicks, R. \& Israel, A.C. (1997). Psicopatología del niño y del adolescente. Madrid: Prentice Hall 